\title{
BREATHING AND CRYING BY NEWLY BORN PRETERM INFANTS IN AN ERA OF DELAYED CORD CLAMPING.
}

\section{INTRODUCTION}

The majority of newly born preterm infants breathe and cry after immediate cord clamping (ICC). ${ }^{1}$ Guidelines recommend delayed cord clamping (DCC) for at least 30 seconds for infants who do not require immediate resuscitation. ${ }^{2}$ Little guidance is given regarding the timing of cord clamping for preterm infants requiring resuscitation. It is not known whether DCC affects the rate of crying and breathing.

\section{AIMS}

To study breathing and crying by extremely preterm infants after cord clamping in an era of DCC

\section{METHODS}

- Observational study

- Tertiary maternity centre (NMH)

- Videos of infants $<28$ weeks or $<1000 g$ (10.16-03.18)

- Aim for cord clamping at $>60$ secs (CPAP/PPV not provided)

- Determined

- Time of birth

- Time infant arrived to resuscitaire (proxy for timing of cord clamping)

- Presence of audible cry before respiratory support

- Presence of visible breathing before respiratory support

- Data analysed with SPSS

- Ethical approval and parental consent

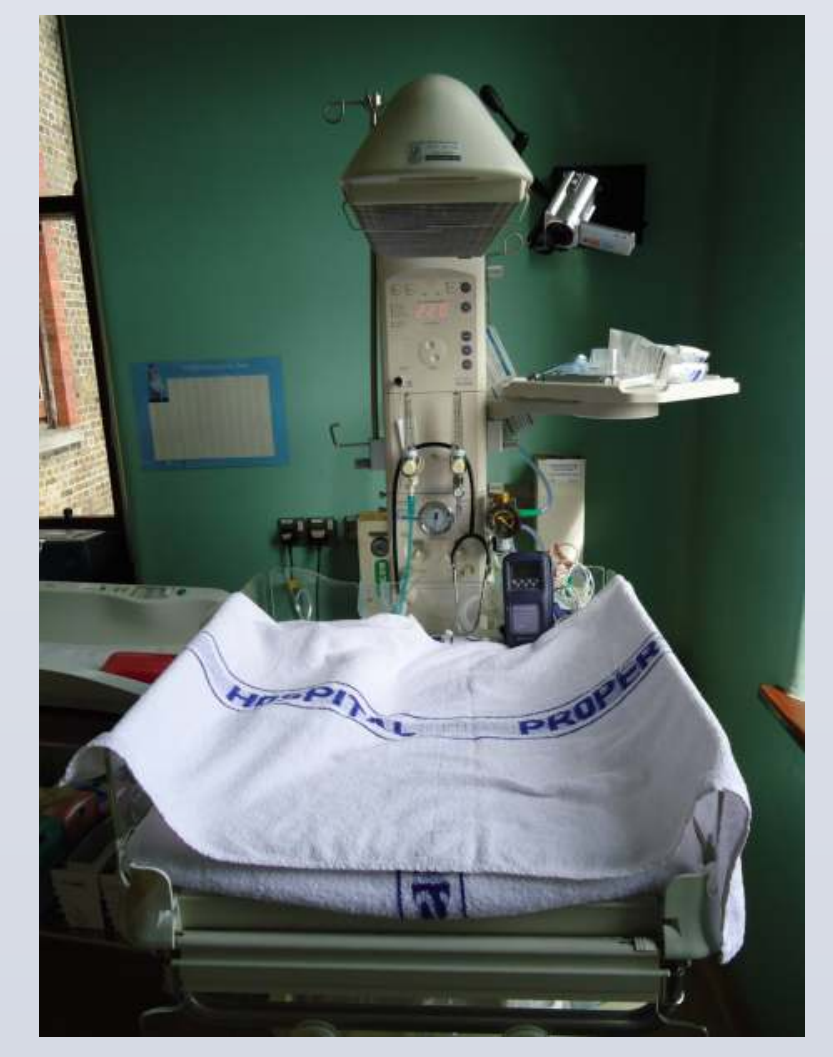

Figure 1. Digital video camera attached to resuscitaire using tripod arm

\section{RESULTS}

- 109 videos recorded between October 2016 \& March 2018

- 35 of eligible infants [mean (SD) GA 27 (2) weeks, BW 890 (200) g]

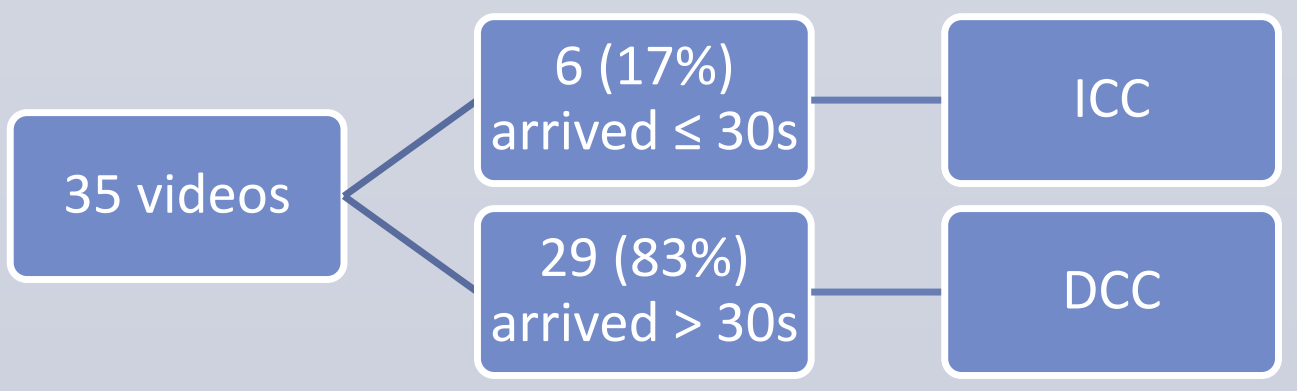

\begin{tabular}{|l|c|c|}
\hline & $\begin{array}{c}\text { ICC } \\
\text { N=6 }\end{array}$ & $\begin{array}{c}\text { DCC } \\
\text { N=29 }\end{array}$ \\
\hline GA, mean (SD), wk & $26(1.6)$ & $27(1.5)$ \\
\hline $\begin{array}{l}\text { BW, mean (SD), g } \\
\begin{array}{l}\text { Arrival to resuscitaire, } \\
\text { mean (SD), s }\end{array}\end{array}$ & $24(8)$ & $81(10)$ \\
\hline
\end{tabular}

Figure 2. Patient characteristics at study entry

Immediate cord clamping $\leq 30 \mathrm{~s}$

\begin{tabular}{|l|c|c|c|}
\hline No. (\%) & Cried & Breathed & $\begin{array}{c}\text { Neither breathed } \\
\text { nor cried }\end{array}$ \\
\hline Total $n=6$ & $4(67)$ & $6(100)$ & 0 \\
\hline
\end{tabular}

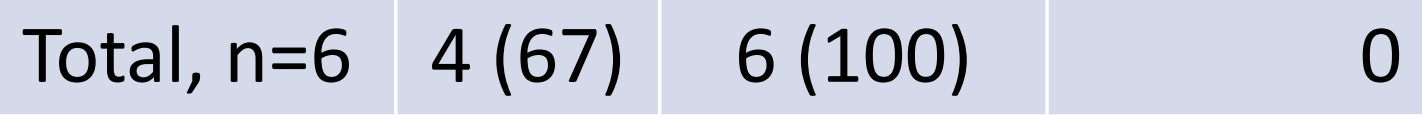

Delayed cord clamping $>30$ s

\begin{tabular}{|l|c|c|c|}
\hline No. (\%) & Cried & Breathed & $\begin{array}{c}\text { Neither } \\
\text { breathed } \\
\text { nor cried }\end{array}$ \\
\hline Total, $\mathrm{n}=29$ & $20(69)$ & $26(90)$ & $3(10)$ \\
\hline GA $\geq 26, \mathrm{n}=21$ & $15(71)$ & $18(86)$ & $3(14)$ \\
\hline GA $<26, \mathrm{n}=8$ & $5(63)$ & $8(100)$ & $0(0)$ \\
\hline BW $\geq 750 \mathrm{~g}, \mathrm{n}=23$ & $17(74)$ & $22(96)$ & $1(4)$ \\
\hline BW $<750 \mathrm{~g}, \mathrm{n}=6$ & $3(50)$ & $4(67)$ & $2(33)$ \\
\hline
\end{tabular}

Subsequent DR support

- Respiratory support given by mask to all 35 infants

- Five (14\%) infants were intubated in the DR

- $2 / 6$ infants who arrived $\leq 30 \mathrm{~s}$

- $3 / 29$ infants who arrived $>30$ s

- Thirty (86\%) infants transferred to NICU on CPAP

\section{CONCLUSIONS}

- Most extremely preterm infants cried and breathed after DCC before respiratory support was given

\section{REFERENCES}

1. O'Donnell CP KC, Davis PG, Morley CJ. Crying and breathing by extremely preterm infants immediately after birth. The Journal of pediatrics. 2010;156(5):846-847.

2. Wyllie J, Perlman JM, Kattwinkel J, et al. Part 7: Neonatal resuscitation: 2015 International Consensus on Cardiopulmonary Resuscitation and Emergency Cardiovascular Care Science with Treatment Recommendations. Resuscitation. 2015;95:e169-201. 